\title{
The impact of laughter yoga on subjective well-being: A pilot study
}

\author{
Melissa K. Weinberg, PhD \\ Thomas G. Hammond, \\ Robert A. Cummins
}

School of Psychology, Faculty of Health, Deakin University, Australia

\begin{abstract}
Laughter has been associated with a number of physiological and psychological benefits. Laughter yoga $(L Y)$ is a contemporary technique that encourages participants to mimic the act of laughing, with the goal of achieving positive psychological outcomes. This paper describes an exploratory pilot study to evaluate the effect of a single LY class on the psychological well-being of people who attended such sessions voluntarily. Forty-four participants $(72.7 \%$ female, Mean age $=58.86, S D=14.12)$ were recruited from $L Y$ clubs around Melbourne, Australia, and completed measures of well-being were taken before and after the LY class. Following the LY session, significant improvements in positive emotions and reductions in the severity of symptoms of anxiety and stress were reported. Importantly, the change in well-being was greatest for participants who were experiencing lower wellbeing prior to the class. These findings suggest that LY shows promise as an effective intervention to temporarily increase well-being.
\end{abstract}

Keywords: subjective well-being; laughter yoga; mood.

\section{Introduction}

Laughter, in its basic form, is a psychophysiological response to a stimulus that leads to the production of muscle contractions, facial expressions, and other neurophysiological processes (Bennett \& Lengacher 2008; Mora-Ripoll 2010; Mora-Ripoll \& Garcia-Rodera 2008). In her review of the benefits of laughter, Mora-Ripoll (2010) concluded that the physiological benefits include improved respiration, general muscle relaxation, and enhanced mental functioning. The physiological changes were accompanied by psychological benefits, including increased positive mood and decreased stress. 
Typically, laughter has been investigated in terms of its ability to reduce negative emotions like anxiety, stress and depression (Houston, McKee, Carroll \& Marsh 1998; MoraRipoll 2010; Papousek \& Schulter 2008; Shahidi et al. 2011). This property was used to propose a stress-buffering hypothesis by Kuiper \& Martin (1998), following their revelation that individuals who reported more frequent laughter experienced less negative affect during stress. In confirmation, Ko \& Youn (2011) reported that laughter therapy reduced depressive symptoms and increased sleep quality in a group of elderly people with high rates of depressive vulnerability.

In addition to reducing negative emotions, unsurprisingly, laughing also increases positive emotions (Mannell \& McMahon 1982) more than simply smiling (Neuhoff \& Schaefer 2002). In his review of the functions of laughter, Askenasy (1987) noted that, while laughing, a person feels free from their cares and worries and experiences prevailing joy. For people who experience more frequent bad moods, laughing may be a good technique to alleviate their negative emotions and restore their well-being to a 'happier' state. Although people in bad moods may not feel as if they have reason to laugh, there is emerging evidence that the laughter does not have to be genuine to elicit positive effects.

In one study, Neuhoff \& Schaefer (2002) instructed participants to either laugh, smile, or howl for one minute, and measured their mood before and after each intervention. Their findings revealed that forced laughter enhanced mood more than either forced smiling or howling. In a subsequent study, Papousek \& Schulter (2008) used the principles of conditioning to promote Cheerfulness training. Through this intervention, annoying or unpleasant experiences were paired with induced cheerful mood to reduce the adverse effects and encourage re-association with positivity. This study was unique as it provided a therapeutic behavioural framework by which to explain how the induction of positive emotions is effective.

Most recently, Ko \& Youn (2011) conducted a laughter therapy programme with community-dwelling elderly people in South Korea. The initial laughter therapy session followed a sequence of relaxation, laughing, clapping, and laughter meditation, and subsequent sessions included dancing and singing to encourage laughter, as well as other laughter exercises. Mean depression scores were reduced for the laughter therapy group following the intervention, whereas no change was evident for the control group. Though the change in depression scores for the laughter therapy group achieved statistical significance, the reduction was just a single point on a 15-point scale. It should also be noted that the depression scores of both groups were elevated at baseline and so the effect of laughter therapy may only apply to those already experiencing low mood levels. Together, these findings provide evidence that forced laughter may be effective at improving positive mood, particularly for those who may be experiencing a reduction in such mood initially.

Laughter yoga (LY) is a specific type of laughter therapy that was developed in India in 1999 by Dr Madan Kataria, and is predicated upon the proposition that simulated laughter can elicit the same physiological and psychological benefits as authentic laughter. Thus, similar to the laughter therapy used in Ko \& Youn's (2011) study, people who cannot achieve genuine laughter may benefit by mimicing the overt behaviour of laughter.

In confirmation of this proposition, the few available studies show LY to be promising as a form of therapy. A 2011 study revealed that elderly depressed women who engaged in LY reported increased life satisfaction compared to exercise therapy and control groups, though they failed to show a significant reduction in depressive symptoms (Shahidi et al. 2011). In other recent studies, patients awaiting organ transplants reported increased feelings of activation and cheerfulness following an LY intervention compared to a control group (Dolgoff-Kaspar et al. 2012), while a South African study of stroke survivours reported increased positive emotions following LY (Suraj-Narayan \& Surajnarayan 2011). Similar 
findings have been reported in an organisational context. Employees who completed 15 sessions of laughter yoga reported significant improvements in perceived self-efficacy in the workplace, and experienced sustained positive emotions at a 90-day follow-up (Beckman, Regier \& Young 2007).

While these results provide evidence to support the use of laughter yoga as a psychological intervention, they have focused largely on clinical samples and have generally neglected to provide a theoretical framework by which to understand how LY can be an effective agent of change. Further, with the increased adoption of LY in mainstream society, no study has as yet explored the effects of LY for people who attend LY clubs at their own general interest and leisure. The present study seeks to explore the effects of LY in a nonclinical, self-selected sample, and provide a theoretical foundation to explain how LY affects well-being. With this understanding, the benefits of LY for well-being may inform future, larger-scale studies.

The homeostasis theory of subjective well-being (Cummins 2010) offers a sound framework within which to explore the effects of laughter yoga on both positive and negative emotions. Subjective well-being (SWB) refers to an individual's perception of the quality of their life, and is primarily driven by an underlying positive and stable, trait-like mood (Cummins 2010). Accordingly, the capacity of laughter yoga to affect subjective well-being is best understood as being a by-product of its influence on positive mood.

Specifically, homeostasis theory holds that SWB is generally held stable and maintained by bio-psychological mechanisms, analogous to the way that body temperature is managed by autonomic systems. As such, there is a normal level of SWB for each person that defines their set-point, and people only become consciously aware of their well-being when some external stressor threatens the system. Using data collected over a period of 12 years, the normative range for sample mean scores for SWB in Australia is found between 73.6 to 76.6 points on a 0-100 point scale (Cummins et al. 2012).

A key implication of homeostasis theory is that there is a 'ceiling effect', whereby SWB cannot chronically be raised above an individual's set-point-range. Accordingly, the effect of laughter yoga on SWB should be greater for those with SWB scores found below the normal range, when compared to those who are at normal functioning levels. A second implication is that if people function below their normal range, they are more likely to seek ways to increase their well-being than are people with normal functioning. Quite simply, there is no need for those already functioning as normal to enhance their well-being. Following this logic, one reason why people may frequent laughter yoga classes in their spare time could be because they function below the normal range of well-being and are seeking resolution to a happier state.

Based on this theoretical framework, the purpose of this study was to examine the effects of a laughter yoga class on SWB. Given the high prevalence rates of depression and anxiety in Australia, an easily accessible and cost-effective intervention that has the potential to reduce negative emotions and increase positive emotions would be highly desirable. As a pilot study, the findings of this research have the potential to inform larger studies, by providing a basis for understanding how laughter yoga affects SWB. It was hypothesised that participants who attended laughter yoga clubs would be functioning at lower levels of wellbeing than the general Australian population before their participation in the LY class. It was also hypothesised that LY would be associated with increases in positive emotions, and reductions in negative emotions. Finally, it was hypothesised that the change in SWB would be greater for those initially functioning below the normal well-being range. 


\section{Method}

\subsection{Participants}

A sample of 44 participants was recruited from seven different Laughter Yoga clubs around Melbourne. Preliminary analyses revealed no differences in initial or subsequent well-being scores based upon which LY club participants attended. The participants ranged in age from $18-87$, with a mean age of $58.86(\mathrm{SD}=14.12)$. Most were female $(72.7 \%)$.

A comparative sample of 44 participants was randomly extracted from the $23^{\text {rd }}$ longitudinal survey of the Australian Unity Well-being Index (Cummins \& Weinberg 2012). This sample was of comparative age (Mean $=59.50, \mathrm{SD}=13.85)$ though it contained a more even spread of males (47.7\%) to females (52.3\%). Since there was no evidence of gender differences for the well-being variables in the data file of origin, the sample was considered an appropriate comparison group.

\subsection{Materials}

Participants completed a questionnaire that included scales to measure general life satisfaction, subjective well-being, and general positive mood. Negative emotions as depressed mood, stress, and anxiety were also included. In addition, demographic information was collected as part of the Time 1 questionnaire.

General Life Satisfaction (GLS): GLS was measured by the single item "How satisfied are you with your life as a whole?". Participants rated their level of satisfaction on an 11-point scale anchored by the terms "Not satisfied at all" to "Completely satisfied".

Subjective Well-being (SWB): SWB was assessed using the Personal Well-being Index (PWI; IWBG 2006), a seven-item measure that approximates general life satisfaction. The seven items represent sub-domains of life as standard of living, health, achieving in life, relationships, personal safety, community connectedness, and future security. Each domain contributes unique statistical variance when regressed against GLS. Participants used the same response scale as for GLS. The PWI has strong psychometric properties and the IWBG report adequate test-retest reliability with Cronbach's alpha ranging between .70 and .85 . Convergent validity has also been established with the Satisfaction with Life Scale (Diener, Emmons, Larsen \& Griffin 1985), giving a correlation of .78 (IWBG 2006).

Mood: General positive mood was measured by asking participants how happy, content, and alert they generally feel on an end-defined 11-point scale from 0 (not at all) to 10 (extremely). These three affective adjectives were selected following findings that showed the terms happy, content, and alert explained in excess of 59\% of variation in SWB and, thus, best represent HPMood, the type of mood that underlies evaluations of Subjective Well-being (Davern, Cummins \& Stokes 2007; Blore, Stokes, Mellor, Firth \& Cummins 2011). Responses to these three items were summed together to obtain an overall HPMood score.

Depression, Anxiety, Stress: The Depression Anxiety Stress Scale (DASS) was used to assess self-reported depressed mood, anxiety and stress. The DASS has demonstrated adequate psychometric properties with the scale authors reporting Cronbach's alpha for the subscales ranging from .84 to .91 . Participants rated how much each statement applied to them on an 11-point scale ranging from "Not at all" to "Extremely".

\subsection{Procedure}

Participants in Laughter Yoga clubs were approached during one of their weekly classes and invited to participate in the study. The nature of the study was explained to them and their 
confidentiality was assured. Members of the research team returned to the LY club the following week and distributed the initial questionnaire for completion prior to the class (Time 1). Participants were instructed to generate a unique code to ensure anonymity but enable their responses to be matched across the different time points. The Time 2 questionnaire was distributed immediately after the end of the Laughter Yoga class and the questionnaires were returned directly to the research team member upon completion.

The intervention followed the standard structure of a Laughter Yoga session, which involves repetition of three key components: During the 'Laugh' phase, the instructor guides participants through activities to simulate laughter. At the beginning of the session, this usually involves a greeting laugh, whereby participants greet each other by shaking hands and laughing while maintaining eye contact. Following this introduction, the 'Laugh' phase can involve different types of laughing, like the "lion laugh", the "telephone laugh" or the "eating breakfast laugh". In the 'Clap' phase of the class, participants pause to repeat the Laughter Yoga mantra of "Ho, ho, ha, ha, ha" while clapping in rhythm. This is followed by the 'Breathe' phase, where participants focus on relaxing and regulating their breathing. Although laughter games and the time spent in each phase can vary class by class, the 'Laugh, Clap, Breathe' structure is common to all sessions. Each session was 30 minutes in duration.

\subsection{Data cleaning and preparation}

Data were screened through SPSS Version 21 and checked for missing values. Cases were only included in analyses if they recorded data at both time points. Within each time point, missing values for individual scale items were found to be random and cases were excluded pair-wise where appropriate, following Pallant (2007). Data were screened for outliers at each time point.

One participant recorded the maximum possible score for SWB and HPMood. This was suggestive of an acquiescent response style and the case was removed from the data set prior to analyses. All scores were converted to percentage of Scale Maximum scores (\%SM) as described in the PWI manual. This procedure transforms all results to lie on a $0-100$ point scale.

\section{Results}

To test the first hypothesis, i.e. that participants who attended Laughter Yoga clubs of their own interest would be functioning at a lower level than the general Australian population, measures captured at Time 1 were compared to a random sample of 44 participants extracted from the $23^{\text {rd }}$ longitudinal survey of the Australian Unity Well-being Index (Cummins \& Weinberg 2012). Within this sample, one participant was identified as having outlying scores on multiple variables, and was excluded from analyses. Comparative data were available for all of the well-being variables, and results are shown in Table 1.

Table 1. Comparison of well-being variables between LY club participants (at Time 1) and a general Australian sample

LY Clubs@ Time 1 General Australian pop.

\begin{tabular}{lllllll}
\hline Variable & Mean & SD & Mean & SD & Comparison & Effect size $(d)$ \\
GLS & 68.60 & 21.56 & 78.57 & 14.24 & $\mathrm{t}(73)=2.521, \mathrm{p}=.014 .55$
\end{tabular}


European Journal of Humour Research1(4)

$\begin{array}{lcccccc}\text { SWB } & 71.95 & 17.17 & 76.55 & 14.34 & \mathrm{t}(80)=1.317, \mathrm{p}=.192 & .29 \\ \text { HPMood } & 70.00 & 19.59 & 75.35 & 15.19 & \mathrm{t}(84)=1.415, \mathrm{p}=.161 & .31 \\ \text { Depression } & 25.02 & 22.85 & 14.29 & 19.09 & \mathrm{t}(84)=2.365, \mathrm{p}=.020 & .51 \\ & & & & & & \\ \text { Anxiety } & 20.16 & 18.19 & 11.03 & 16.04 & \mathrm{t}(83)=2.456, \mathrm{p}=.016 & .53 \\ & & & & & & \\ \text { Stress } & 35.23 & 25.26 & 22.56 & 17.47 & \mathrm{t}(71)=2.661, \mathrm{p}=.010 & .58\end{array}$

The figures in Table 1 reveal that the participants who frequented LY clubs reported lower general life satisfaction, with a GLS score of 68.60 being well below the normative range of 76.02 to 79.24 points (Table A2.21, Cummins et al. 2012). Similarly, their SWB score of 71.95 was well below the normal range of 73.78 to 76.68 points. The comparison sample, on the other hand, had a mean score on both variables that lay within their respective normal ranges. Although the difference between groups for SWB and HPMood was not large enough to achieve statistical significance, the small to medium effect size reported indicates that these trends would likely achieve significance in a larger sample (Cohen 1988).

The LY group members also had higher rates of negative emotions (depressed mood, anxiety, and stress) than the general Australian sample. The effect size was highest for the difference in stress levels between the two samples.

The DASS Manual (Lovibond \& Lovibond 1995) provides suggested cut-off scores for diagnoses of levels of each of depression, anxiety, and stress. These are based on scores out of a possible 42, and following conversion to percentage of scale maximum scores, the LY group in this study fall within the 'mild' categories on each construct. The general Australian sample, on the other hand, is considered 'normal' by these cut-off scores.

To test the second hypothesis, i.e. that participation in a Laughter Yoga class would be associated with increases in SWB and decreases in negative emotions, repeated-measures ttests were conducted to compare scores at Time 1 and Time 2 for LY participants.

Table 2. Comparison of well-being variables pre- and post-LY class

\begin{tabular}{llllllll}
\hline \multicolumn{7}{c}{ Time 1 } & Time 2 \\
\hline Variable & Mean & SD & Mean & SD & Comparison & Effect size $(d)$ \\
GLS & 68.60 & 21.56 & 77.21 & 16.08 & $\mathrm{t}(42)=3.873, \mathrm{p}=.000$ & .40 \\
SWB & 71.93 & 17.39 & 77.96 & 12.14 & $\mathrm{t}(39)=3.437, \mathrm{p}=.001$ & .35 \\
HPMood & 70.00 & 19.59 & 75.12 & 18.39 & $\mathrm{t}(42)=2.159, \mathrm{p}=.037$ & .26 \\
Depression & 23.87 & 22.40 & 22.86 & 20.30 & $\mathrm{t}(40)=.556, \mathrm{p}=.581$ & .05 \\
Stress & 35.68 & 25.42 & 30.61 & 24.01 & $\mathrm{t}(39)=2.347, \mathrm{p}=.024$ & .20 \\
Anxiety & 20.23 & 18.41 & 16.41 & 16.61 & $\mathrm{t}(40)=2.440, \mathrm{p}=.019$ & .21
\end{tabular}

Table 2 reveals that participation in a Laughter Yoga class was associated with an immediate improvement in general life satisfaction and subjective well-being. The value for GLS now fell within the normal range, while SWB was above its normal range. While statistically 
significant changes were also apparent in mood, stress, and anxiety, these levels of significance are marginal after the application of a Bonferroni correction. The largest effect was apparent for general life satisfaction, and there was no effect of LY to reduce symptoms of depression.

To determine whether participation in Laughter Yoga classes would be more beneficial for participants who reported lower levels of SWB, participants were split according to their SWB scores at Time 1. A cut-off score of 75.00 was used to split the sample into 2 groups, as 75.00 is the mean score for the Australian population (Cummins et al. 2012). A change score for SWB was computed as the Time 2 score minus the Time 1 score. Comparison of the change scores for each group revealed that the magnitude of change was significantly greater for participants initially reporting lower SWB $(\mathrm{M}=10.29, \mathrm{SD}=13.67)$ than participants who were functioning within the normal range $(\mathrm{M}=1.79, \mathrm{SD}=5.32), t(25)=2.592, \mathrm{p}<.05$.

An indicator of reliability change is useful, in this instance, to provide an indication as to whether the change in SWB scores is practically significant beyond what may be due to unreliability of the measure. The Reliability Change Index (Jacobson \& Truax 1991) was calculated; it revealed that over a third of participants in the study achieved a change in SWB score larger than two standard deviations. Further, four times as many participants who achieved a reliable change reported SWB scores below 75 at Time 1 . These results provide preliminary evidence to support the effectiveness of laughter yoga as a form of therapy to increase SWB for those whose SWB is below the normal range, but require validation in a larger study.

\section{Discussion}

Grounded within homeostasis theory, the present study aimed to explore the effects of Laughter Yoga (LY) on Subjective Well-being (SWB) to guide future research in this area. Given the small sample size and absence of a genuine control group, the interpretations made herein are only suggestive, and all findings require further testing and validation before they can be generalised to a wider sample.

Prior to the start of the LY session, it was observed that individuals who attended the LY classes were found to report levels of General Life Satisfaction (GLS) and SWB below normative ranges for the Australian population and to also have higher levels of depression, anxiety, and stress. These findings lend support to the implications of homeostasis theory (Cummins 2010), and the notion that people functioning below the normal range of wellbeing are more likely to be aware of their affective state, and actively seek out opportunities to improve their well-being.

Following the conclusion of the single LY session, there were improvements in SWB and GLS scores. Mood also increased, though not to the same extent. The finding that positive emotions increase following an induced laughter intervention is consistent with previous research (Dolgoff-Kaspar et al. 2012; Suraj-Narayan \& Surajnarayan 2011; Beckman, Regier \& Young 2007). Specifically, the increased GLS is consistent with the findings of Shahidi et al. (2011) who found that elderly participants diagnosed with depression also reported increases in GLS. The present study extended these findings, and indicated that a single LY session is associated with increased GLS even for people who are functioning below normal, but have not achieved diagnostic levels of psychopathology.

Following the single LY class, anxiety and stress scores were reduced from mild severity ratings to the normal range based on suggested DASS classifications (Lovibond \& Lovibond 1995). However, depression levels remained in the mild range. The statistically significant reduction in anxiety and stress symptoms post-LY may have been related to 
physiological changes associated with laughter. The muscle relaxation, reduced heart rate, and improved circulation associated with the induction of laughter (Mora-Ripoll 2010), may have alleviated the somatic symptoms associated with anxiety and stress. Depression, however, is not only characterised by low mood but is also associated with maladaptive cognitions, anhedonia and inertia (Lovibond \& Lovibond 1995). As such, although inducing laughter may have the capacity to facilitate improvements in affective states, it may be insufficient to address the cognitive dysfunction and other symptoms that typify depression.

The final hypothesis, i.e. that larger increases in SWB would be observed for participants initially functioning below the normal level, was also supported. The increase in scores on SWB for participants who scored below the 'gold-standard' of 75.00 prior to the LY class was five times higher than for those who were already functioning above 75.00. Further, the change scores for participants who reported SWB below 75.00 reached the clinical reliability standard much more frequently than for those who reported SWB at or above the gold-standard at Time 1. This finding lends support to homeostasis theory (Cummins 2010), and is suggestive of important practical implications for the effectiveness of programmes intended to increase SWB. There appears to be a ceiling effect for SWB. As such, the findings of this study suggest that interventions aimed at improving SWB will be most advantageous for people who are functioning below the optimal level, and are in need of assistance to regain normal functioning. Those people who are operating within the normal range will experience little benefit. This is an important caveat for positive psychology interventions as the potential for increased well-being is not universal. The effectiveness of interventions to consistently increase SWB warrants further investigation with larger samples that include clinical and nonclinical participant groups.

While this study has provided interesting information, its usefulness would be enhanced by longer-term investigations that evaluated the changes in SWB over time. Certainly, of interest would be the duration of the positive effects following LY sessions. In the present study, participants were not attending their LY class for the first time and, thus, the Time 1 score is not a true indicator of baseline functioning. In fact, it implies that the effect of LY is not long-lasting, as participants returning for subsequent classes report lower SWB scores than they did following the LY class. In addition, the present study was unable to control the exact structure of each LY class, so the intervention administered at each location was not identical. It is plausible that there might be some aspects of LY that are more conducive to improving well-being, but were not practised routinely in each different class.

Further, it remains possible that, since participants were attending the LY session with the implied intention of increasing their SWB, they would be more likely to endorse an increase in well-being following the class. This may be of particular concern given the short time frame between the pre- and post-questionnaires. However, the finding that initial wellbeing scores were below the normal range for Australians suggests that these were true scores recorded by the sample, as it is extremely unlikely that they would have had knowledge of the normal ranges, and so could not have intentionally recorded their scores to be lower. Further, if recall effects were evident, then they would be expected to be expressed across each measure. The lack of change in depression scores suggests that participants were not motivated to report that LY was advantageous across the board of measures used.

Finally, the presence of a suitable control group could confirm that the improvement in well-being variables was due to the intervention, rather than representing the statistical phenomenon of regression to the mean. The inclusion of a control group would also enable consideration of whether laughter yoga, as a specific intervention, is more effective at improving SWB than other interventions. The findings of this study do not provide support for the effectiveness of LY over and above any other form of therapy, and it remains unknown 
whether the improvements to SWB are due to the intervention itself, or due to participants' benefiting from the social environment that LY classes facilitate.

Despite the clear need for a control group, an appropriate control group is difficult to identify given that LY is such a unique activity. A suitable control group activity should be similar in all aspects to the LY group except for the specific practice of laughter yoga, but there is no conceivable alternative. For example, although participants undertaking regular yoga sounds like an obvious comparison group, the aim of regular yoga is to reduce heart rate and encourage relaxation, whereby the act of laughing necessarily increases heart rate. In the absence of a suitable control group, the Australian normative data were considered an appropriate comparison for this study.

While the benefits of laughter to improve positive emotions and reduce negative emotions are difficult to contend, the results of this study caution that the effectiveness is not universal. People already experiencing a normal level of well-being may not only be less likely to attend LY, but also appear to have less to gain from them. Thus, LY, similar to other positive psychology techniques, is perhaps best described as an intervention more likely to benefit people experiencing lower levels of SWB, and higher levels of anxiety and stress. This study has provided preliminary, albeit cautionary, results for the effectiveness of LY as an intervention to increase SWB, and requires further validation in a larger scale study.

\section{Notes}

* All correspondence should be addressed to Melissa K. Weinberg, School of Psychology, Faculty of Health, Deakin University, Australia: melissa.weinberg@deakin.edu.au

\section{References}

Askenasy, J. J. M. (1987). 'The functions and dysfunctions of laughter'. Journal of General Psychology 114, pp. 317-334.

Beckman, H., Regier, N. \& Young, J. (2007). 'Effect of workplace laughter groups on personal efficacy beliefs'. The Journal of Primary Prevention 28, pp. 167-182.

Bennett, M. P. \& Lengacher, C. (2008). 'Humour and laughter may influence health - III: Laughter and health outcomes'. Evidence-based Complementary and Alternative Medicine 5, pp. 37-40.

Blore, J. D., Stokes, M. A., Mellor, D., Firth, L. \& Cummins, R. A. (2011). 'Comparing multiple discrepancies theory to affective models of subjective well-being'. Social Indicators Research 100, pp. 1-16.

Cohen, J. (1988). Statistical Power Analysis for the Behavioural Sciences. (2 ${ }^{\text {nd }}$ edition). Hillsdale, NJ: Erlbaum.

Cummins, R. A. (2010). 'Subjective well-being, homeostatically protected mood and depression: A synthesis'. Journal of Happiness Studies 11, pp. 1-17.

Cummins, R. A. \& Weinberg, M. K. (2012). Australian Unity Well-being Index: Longitudinal Survey ARC23. Melbourne: Deakin University.

Cummins, R. A., Woerner, J., Weinberg, M. K., Collard, J., Hartley-Clark, L., Perera, C. \& Horfiniak, K. (2012). Australian Unity Well-being Index: Report 28.0 - The Well-being of Australians: The Impact of Marriage. Melbourne: Australian Centre on Quality of Life.

Davern, M. T., Cummins, R. A. \& Stokes, M. A. (2007). 'Subjective well-being as an affective-cognitive construct'. Journal of Happiness Studies 8, pp. 429-449.

Diener, E., Emmons, R. A., Larsen, R. J. \& Griffin, S. (1985). 'The satisfaction with life scale'. Journal of Personality Assessment 49, pp. 71-75. 
Dolgoff-Kaspar, R., Baldwin, A., Johnson, S., Edling, N. \& Sethi, G. K. (2012). 'Effect of Laughter Yoga on mood and heart rate variability in patients awaiting organ transplantation: A pilot study'. Alternative Therapies in Health \& Medicine 18, pp. 61-66.

Houston, D. M., McKee, K. J., Carroll, L. \& Marsh, H. (1998). 'Using humour to promote psychological well-being in residential homes for older people'. Aging \& Mental Health 2, pp. 328-332.

International Well-being Group (2006). Personal Well-being Index. Melbourne: Australian Centre on Quality of Life, Deakin University.

Jacobson, N. S. \& Truax, P. (1991). 'Clinical significance: A statistical approach to defining meaningful change in psychotherapy research'. Journal of Consulting and Clinical Psychology 59, pp. 12-19.

Ko, H. J. \& Youn, C. H. (2011). 'Effects of laughter therapy on depression, cognition and sleep among the community-dwelling elderly'. Geriatrics \& Gerontology International 11, pp. 267-274.

Kuiper, N. A. \& Martin, R. A. (1998). 'Laughter and stress in daily life: Relation to positive and negative affect'. Motivation and Emotion 22, pp. 133-153.

Lovibond, S. H. \& Lovibond, P. F. (1995). Manual for the Depression Anxiety Stress Scales. ( $2^{\text {nd }}$ edition). Sydney: The Psychology Foundation of Australia Inc.

Mannell, R. C. \& McMahon, L. (1982). 'Humour as play: Its relationship to psychological well-being during the course of a day'. Leisure Sciences 5, pp. 143-155.

Mora-Ripoll, R. (2010). 'The therapeutic value of laughter in medicine'. Alternative Therapies in Health \& Medicine 16, pp. 56-64.

Mora-Ripoll, R. \& Garcia-Rodera, N. C. (2008). 'Therapeutic value of laughter in medicine'. Med Clin (Barc) 131, pp. 694-698.

Neuhoff, C. C. \& Schaefer, C. (2002). 'Effects of laughter, smiling, and howling on mood'. Psychological Reports 91, pp. 1079-1080.

Pallant, J. (2007). SPSS Survival Manual: A Step by Step Guide to Data Analysis Using SPSS for Windows. ( $3^{\text {rd }}$ edition). NSW: Allen \& Unwin.

Papousek, I. \& Schulter, G. (2008). 'Effects of a mood-enhancing intervention on subjective well-being and cardiovascular parameters'. International Journal of Behavioural Medicine 15, pp. 293-302.

Shahidi, M., Mojtahed, A., Modabbernia, A., Mojtahed, M., Shafiabady, A., Delavar, A. \& Honari, H. (2011). 'Laughter yoga versus group exercise program in elderly depressed women: A randomized controlled trial'. International Journal of Geriatric Psychiatry 26, pp. 322-327.

Suraj-Narayan, G. S. \& Surajnarayan, S. (2011). Biopsychosocial Impacts of Laughter Yoga and Therapy on Stroke Survivours. South Africa: African Laughter Institute. 